\title{
2003 Survey of the Florida Caladium Tuber Production Industry 1
}

\section{Z. Deng, B.K. Harbaugh, R.K. Schoellhorn, and R.C. Andrews ${ }^{2}$}

Caladiums are popular tropical plants because of their bright colorful leaves and tolerance to both heat and shade. Potted and landscape caladium plants are grown from tubers (they are often called bulbs). Most of the tubers (over 90\%) used in the world are produced by Florida caladium growers in Lake Placid and Sebring, Highlands County. These growers and their tuber production industry have been an important component of the state's environmental horticulture industry and have a worldwide impact.

A survey of the Florida caladium growers and industry was conducted from June to August 2003. Objectives of the survey were 1) to gather basic demographic information about the Florida caladium tuber production industry, 2) to understand the industry's needs for new varieties, research and informational resources, and 3) to determine caladium cultivars that are currently grown commercially and to identify the leading cultivars. Similar surveys were conducted in 1979 and 1997.

Survey forms were mailed to 20 business operations, of which 14 are currently growing caladiums for tuber production. A total of 13 growers returned their surveys. The responses represented at least $95 \%$ of the total acreage currently in caladium tuber production.

\section{Industry-Wide General Information}

Respondents reported 1258 acres and 1330 acres in caladium tuber production in 2002 and 2003, respectively. These acreages are close to the 1311 acres reported in the 1997 survey. The average number of years in business was 23 years. Growers employed an average of 7 full-time employees plus 11 seasonal part-time workers. Multiple marketing channels are being used by all the responding growers.

\section{Industry Perceptions of the Need for Improved Caladium Cultivars}

Thirteen new cultivars have been released from the University of Florida /IFAS's caladium breeding program at the Gulf Coast Research and Education Center. They were grown on over 130 acres in 2002-2003. The industry indicated a high demand for new cultivars with improved resistance or tolerance to major diseases, especially Fusarium tuber rot and Pythium root rot, and root-knot nematodes. In

1. This document is ENH1007, one of a series of the Environmental Horticulture Department, Florida Cooperative Extension Service, Institute of Food and Agricultural Sciences, University of Florida. Original publication date August 8, 2005. Visit the EDIS Web Site at http://edis.ifas.ufl.edu.

2. Z. Deng, assistant professor; B.K. Harbaugh, professor, Environmental Horticulture Department, Gulf Coast Research and Education Center; R.K. Schoellhorn, associate professor, Environmental Horticulture Department, University of Florida; R.C. Andrews, horticultural extension agent, Highlands County Cooperative Extension Service, IFAS, University of Florida, Gainesville, FL 32611.

The Institute of Food and Agricultural Sciences (IFAS) is an Equal Opportunity Institution authorized to provide research, educational information and other services only to individuals and institutions that function with non-discrimination with respect to race, creed, color, religion, age, disability, sex, sexual orientation, marital status, national origin, political opinions or affiliations. U.S. Department of Agriculture, Cooperative Extension Service, University of Florida, IFAS, Florida A. \& M. University Cooperative Extension Program, and Boards of County Commissioners Cooperating. Larry Arrington, Dean 
addition, improved tuber yield and quality were considered as priority breeding objectives.

\section{Industry Perceptions of Needs in Other Research Areas}

Several areas have been identified as research needs. These areas include: 1) fertilization recommendation as related to the fertilizer analysis, rate, frequency and timing of application; 2) disease management for grassy tubers and leaf blight; 3 ) new herbicides for weed control in production fields; 4) tuber curing, storage, and postharvest physiology; and 5) application of plant growth regulators to replace the costly manual de-eyeing process.

\section{Industry Perceptions of Needs for Information on the Internet}

The majority of respondent growers expressed their interest in being listed on the web pages in the website that is developed at the Gulf Coast Research and Education Center. They encouraged researchers to use the Internet and websites to deliver information related to tuber quality, container forcing, greenhouse production of pot plants, and correct landscape use to brokers, greenhouse growers, wholesale customers, homeowners, and other end users. Promoting the use of caladiums in the landscape is viewed as a high priority.

\section{Caladium Cultivars Grown in Florida}

A total of 108 distinct cultivars were listed in the survey form. Respondent growers identified 92 cultivars as presently being grown (Table 1). Seventy-six and 16 of the currently grown cultivars were fancy and lance-leaved, respectively. The majority of growers each grew 20-30 cultivars while 35 of the 92 cultivars were exclusively grown by a single grower. Over $99 \%$ of the acreage currently utilized by the industry was used to propagate 53 cultivars. The top 10 fancy-leaved cultivars were 'White Christmas' (11.6\% of the total fancy acreage), 'Candidum' (11.0\%), 'Carolyn Whorton' (7.1\%), 'Pink Beauty' (6.6\%), 'Frieda Hemple' (6.4\%), 'Fannie Munson' (5.7\%), 'White Queen' (5.1\%), 'Candidum Jr.' (4.6\%), 'Red Flash' (4.2\%), and 'Postman Joyner' (3.8\%) (Figure 1). The top 10 lance-leaved cultivars were 'Florida Sweetheart' (26.8\% of the total lance acreage), 'White Wing' (15.4\%), 'Red Frill' (15.4\%), 'Florida Red Ruffles' (9.4\%), 'Pink Gem' (9.2\%), 'Lance Whorton' (6.3\%), 'Rosalie' (6.3\%), 'Pink Symphony' (5.9\%), 'Jackie Suthers' (2.6\%), and 'White Knight' (1.3\%) (Figure 2).

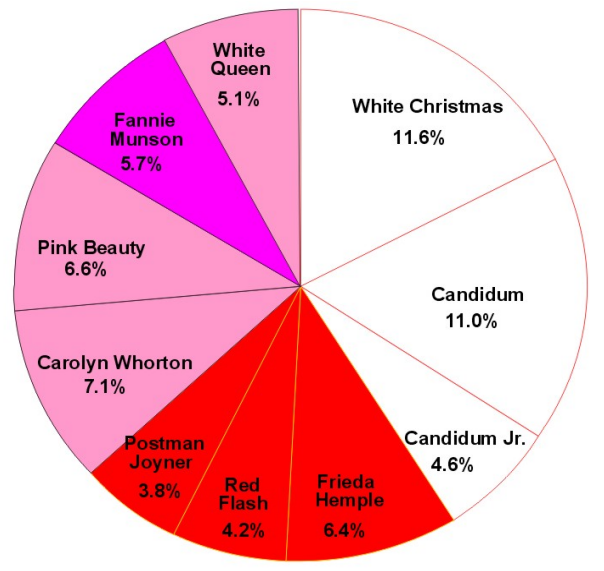

Figure 1. Top 10 fancy cultivars as a percentage of total fancy cultivar acreage. Credits: UF/GCREC

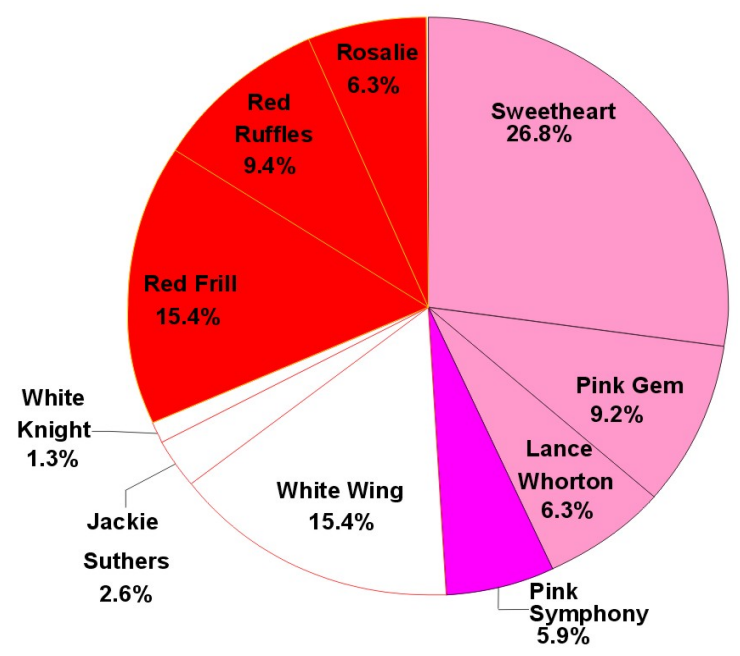

Figure 2. Top 10 lance-leaved cultivars as a percentage of the total lance acreage. Credits: UF/GCREC 


\section{Literature cited}

Bell M.L., G.J. Wilfret, and D.A. DeVoll. 1998.

Survey of caladium tuber producers for acreage of cultivars grown. Proceedings of the Florida State Horticultural Society 111:32-34.

Wilfret, G.J. and G.T. Hurner Jr. 1982. A survey of caladium cultivars grown in Florida and their characteristics as potted plants. Proceedings of the Florida State Horticultural Society 95:190-194. 
Table 1. Acreage and overall rank of caladium cultivars grown in Florida.

\begin{tabular}{|c|c|c|c|c|}
\hline Cultivar & $\begin{array}{l}\text { Leaf } \\
\text { shape }\end{array}$ & Leaf color & Acreage & $\begin{array}{c}\text { Overall } \\
\text { rank }\end{array}$ \\
\hline Aaron & Fancy & White & 40.8 & 12 \\
\hline Ace of Hearts & Fancy & Pink & $<0.1$ & - \\
\hline Apple Blossom & Fancy & Pink & $<0.1$ & - \\
\hline Attala & Fancy & Pink & $<0.1$ & - \\
\hline Autumn Beauty & Fancy & Novelty & $<0.1$ & - \\
\hline Big Red & Fancy & Red & 0.2 & - \\
\hline Blaze (= Dr. T.L. Meade) & Fancy & Red & 9.6 & 34 \\
\hline Brandywine (=Irane Dank) & Fancy & Red & 13.0 & 24 \\
\hline Buck & Fancy & Red & 10.9 & 28 \\
\hline Candidum & Fancy & White & 120.6 & 2 \\
\hline Candidum Jr. & Fancy & White & 50.0 & 8 \\
\hline Carolyn Jane & Fancy & Pink & $<0.1$ & - \\
\hline Carolyn Whorton & Fancy & Pink & 77.7 & 3 \\
\hline Crescent Queen & Fancy & Novelty & $<0.1$ & - \\
\hline Crimson Wave & Fancy & Pink & 0.1 & - \\
\hline Dawn (=Day Break) & Fancy & Pink & 0.1 & - \\
\hline Dr. Groover & Fancy & Pink & 1.0 & 53 \\
\hline Edith Meade & Fancy & Pink & $<0.1$ & - \\
\hline Elizabeth Lou & Lance & Pink & $<0.1$ & - \\
\hline Etta Moore & Lance & Novelty & $<0.1$ & - \\
\hline Fannie Munson & Fancy & Pink & 61.8 & 6 \\
\hline Festivia & Fancy & Red & 2.7 & 44 \\
\hline Fire Chief & Fancy & Pink & 24.3 & 17 \\
\hline Fire Nymph & Fancy & Red & $<0.1$ & - \\
\hline Florida Beauty & Fancy & Pink & 0.6 & - \\
\hline Florida Blizzard & Fancy & White & 0.5 & - \\
\hline Florida Calypso & Fancy & Novelty & 2.5 & 46 \\
\hline Florida Cardinal & Fancy & Red & 37.5 & 13 \\
\hline Florida Elise & Fancy & Pink & 11.6 & 27 \\
\hline Florida Fantasy & Fancy & Novelty & 9.7 & 33 \\
\hline Florida Irish Lace & Lance & Novelty & $<0.1$ & - \\
\hline Florida Moonlight & Fancy & White & 0.2 & - \\
\hline Florida Red Ruffles & Lance & Red & 15.0 & 21 \\
\hline Florida Roselight & Fancy & Pink & 6.5 & 38 \\
\hline Florida Sunrise & Fancy & Novelty & 9.7 & 33 \\
\hline
\end{tabular}


Table 1. Acreage and overall rank of caladium cultivars grown in Florida.

\begin{tabular}{|c|c|c|c|c|}
\hline $\begin{array}{l}\text { Florida Sweetheart } \\
\text { Cultivar }\end{array}$ & $\begin{array}{l}\text { Lance } \\
\text { Leaf } \\
\text { shape }\end{array}$ & $\begin{array}{c}\text { Pink } \\
\text { Leaf color }\end{array}$ & $\begin{array}{c}42.7 \\
\text { Acreage }\end{array}$ & $\begin{array}{c}10 \\
\text { Overall } \\
\text { rank }\end{array}$ \\
\hline Florida White Ruffles & Lance & White & 0.4 & - \\
\hline Florida Whitewater & Lance & White & 1.9 & 48 \\
\hline Fred Bause & Fancy & Red & $<0.1$ & - \\
\hline Frieda Hemple & Fancy & Red & 69.8 & 5 \\
\hline Galaxy & Fancy & White & 1.2 & 51 \\
\hline Gingerland & Fancy & Novelty & 20.2 & 20 \\
\hline Grey Ghost & Fancy & White & 7.1 & 37 \\
\hline Gypsy Rose & Fancy & Pink & 4.1 & 40 \\
\hline Ivory & Fancy & White & $<0.1$ & - \\
\hline Jackie Suthers & Lance & White & 4.1 & 41 \\
\hline Jesse Thayer & Fancy & & $<0.1$ & - \\
\hline John Peed & Fancy & Red & 22.6 & 18 \\
\hline Jubilee & Fancy & Novelty & 0.1 & 30 \\
\hline June Bride & Fancy & White & 9.8 & 32 \\
\hline Kathleen & Fancy & Pink & 10.5 & 29 \\
\hline Lance Whorton & Lance & Novelty & 10.1 & 30 \\
\hline Lee Stokes & Fancy & Pink & $<0.1$ & - \\
\hline Lord Derby & Fancy & Pink & 1.7 & 50 \\
\hline Madam Truall & Fancy & Pink & $<0.1$ & - \\
\hline Marie Moir & Fancy & Novelty & 1.2 & 52 \\
\hline Miss Chicago & Fancy & Pink & 0.1 & - \\
\hline Miss Louisiana & Fancy & Pink & 0.1 & - \\
\hline Miss Muffet & Fancy & Novelty & 22.2 & 19 \\
\hline Mrs. Arno Nehrling & Fancy & Novelty & 14.0 & 23 \\
\hline Mrs. F. Sanders & Fancy & Pink & $<0.1$ & - \\
\hline Mrs. F.M. Joyner & Fancy & Pink & 3.8 & 42 \\
\hline Mrs. W.B. Halderman & Fancy & Pink & 1.7 & 49 \\
\hline New John Peed & Fancy & Red & $<0.1$ & - \\
\hline Pink Beauty & Fancy & Pink & 72.0 & 4 \\
\hline Pink Cloud & Fancy & Pink & 11.9 & 25 \\
\hline Pink Gem & Lance & Pink & 14.6 & 22 \\
\hline Pink Glow & Fancy & Pink & $<0.1$ & - \\
\hline Pink Symphony & Lance & Pink & 9.4 & 35 \\
\hline Poecile Anglais & Fancy & Red & 11.8 & 26 \\
\hline
\end{tabular}


Table 1. Acreage and overall rank of caladium cultivars grown in Florida.

\begin{tabular}{|c|c|c|c|c|}
\hline $\begin{array}{l}\text { Postman Joyner } \\
\text { Cultivar }\end{array}$ & $\begin{array}{l}\text { Fancy } \\
\text { Leaf } \\
\text { shape }\end{array}$ & $\begin{array}{c}\text { Red } \\
\text { Leaf color }\end{array}$ & $\begin{array}{c}41.4 \\
\text { Acreage }\end{array}$ & $\begin{array}{c}11 \\
\text { Overall } \\
\text { rank }\end{array}$ \\
\hline Pothos & Fancy & Novelty & $<0.1$ & - \\
\hline Red Flare & Fancy & Novelty & $<0.1$ & - \\
\hline Red Flash & Fancy & Red & 45.6 & 9 \\
\hline Red Frill & Francy & Red & 24.5 & 16 \\
\hline Rosalie & Lance & Red & 10.0 & 31 \\
\hline Rosebud & Fancy & Pink & 33.8 & 14 \\
\hline Royal White Robe & Fancy & White & $<0.1$ & - \\
\hline Ruby Smith & Fancy & Red & $<0.1$ & - \\
\hline Scarlet Beauty & Fancy & Red & 9.0 & 36 \\
\hline Scarlet Pimpernell & Fancy & Red & 2.8 & 43 \\
\hline Sea Gull & Fancy & White & $<0.1$ & - \\
\hline Texas Beauty & Fancy & Red & 0.1 & - \\
\hline Tom Tomlinson & Fancy & Pink & 5.9 & 39 \\
\hline Torchy & Fancy & Red & 0.5 & - \\
\hline Triumph de L' Exposition & Fancy & Novelty & $<0.1$ & - \\
\hline White Christmas & Fancy & White & 126.6 & 1 \\
\hline White Knight (=Lady of Fatima) & Lance & White & 2.0 & 47 \\
\hline White Princess & Lance & White & $<0.1$ & 1 \\
\hline White Queen & Fancy & Novelty & 56.0 & 7 \\
\hline White Wing & Lance & White & 24.5 & 15 \\
\hline Zo Munson & Fancy & Pink & $<0.1$ & - \\
\hline
\end{tabular}

